\title{
Mathematical modelling of space charge breakdown in membrane systems taking into account the non-catalytic dissociation/ recombination reaction of water molecules
}

\author{
$V$ Gudza $^{1}, M$ Urtenov $^{1,}{ }^{*}, N$ Chubyr ${ }^{2}, I$ Shkorkina ${ }^{1}$ \\ ${ }^{1}$ Kuban State University, Krasnodar 350040, Russia \\ ${ }^{2}$ Kuban State Technological University, Krasnodar 350072, Russia
}

\begin{abstract}
In electromembrane systems, a theoretical study of salt ion transfer usually uses mathematical models of salt ion transfer in the depleted diffusion layer of ion-exchange membranes. In this paper, a new mathematical model of ion transport in the cross-section of the desalination channel formed by two ion-exchange membranes - anion-exchange (AEM) and cation-exchange (CEM), taking into account the non-catalytic dissociation/recombination reaction of water molecules. The model is a boundary value problem for a non-stationary system of Nernst-Planck and Poisson equations. A numerical analysis of the boundary value problem is performed and the main regularities of the 1:1 salt ion transfer process are established, in particular, the occurrence and development of space charge breakdown is shown. The interaction of the space charge and the noncatalytic dissociation/recombination reaction of water molecules are theoretically investigated.
\end{abstract}

\section{Introduction}

Studies $[1,2]$ show the appearance and development of an extended space charge region in electromembrane systems under overlimiting current conditions. We showed theoretically using a mathematical model of 1:1 ion transport of salt ions [3], the occurrence of a space charge breakdown [1], in which the space charge of the cation-exchange membrane (CEM) and the space charge of the anion-exchange membrane (AEM) touch and the space charge of both membranes decreases, but the conduction channel consisting of salt ions is preserved.

Studies [2, 4] shown, that the dissociation/recombination reaction of water molecules occurs in electromembrane systems, which has a significant effect on the processes of salt ion transfer. We have shown $[5,6]$ that a non-catalytic dissociation/recombination reaction of water molecules occurs at overlimiting current densities. The occurrence and development of the space charge and the dissociation/recombination reactions of water molecules that occur under overlimiting current conditions have been studied

\footnotetext{
*Corresponding author: urtenovmax@mail.ru
} 
independently. At the same time, the appearance of new charge carriers during the dissociation of water molecules, namely hydrogen and hydroxyl ions, should strongly affect the formation and development of the space charge and, accordingly, the breakdown of the space charge. This raises an actual problem of theoretical investigation of the effect of dissociation/recombination of water molecules on space charge breakdown. In the article, a mathematical model is formulated in the form of a boundary value problem for the system of Nernst-Planck and Poisson equations, and a numerical analysis of the boundary value problem is performed. The main regularities of the $1: 1$ salt ion transfer process are established, in particular, the occurrence and development of space charge breakdown is shown. The interaction of the space charge and the non-catalytic dissociation/recombination reaction of water molecules on the transport of salt ions are theoretically investigated.

\section{Mathematical model}

Consider a desalination channel formed by anion-exchange and cation-exchange membranes and a width $H$. An aqueous solution of binary salt is fed to the channel entrance. As an example, a solution of potassium chloride is considered. The channel section diagram is shown in Figure 1:

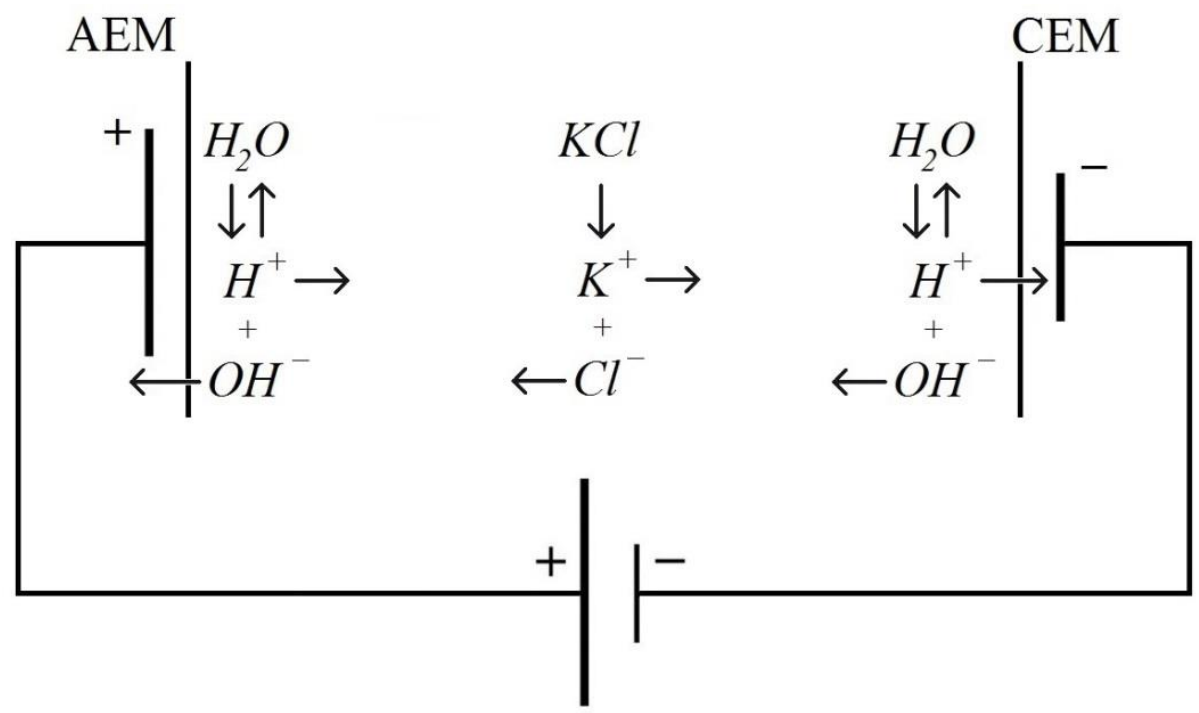

Fig. 1. Scheme for the problem statement of cross-section of the desalination channel formed by AEM and CEM, with the reactions occurring in it.

If the channel is long and narrow enough, and the flow of the solution is small, then the processes occurring in different sections, away from the entrance and exit of the channel, will be almost identical. Therefore, it is sufficient to study the breakdown of the space charge and the dissociation/recombination reaction of water molecules in the cross-section of the desalination channel. The non-stationary transfer of salt ions for 1:1 electrolyte in the cross-section of channel, taking into account the space charge and the dissociation/recombination reaction, is described by the following system of equations [7]:

$$
\frac{\partial C_{i}}{\partial t}=-\frac{\partial j_{i}}{\partial x}+R_{i}, \quad i=1, \ldots, 4
$$




$$
\begin{gathered}
j_{i}=-z_{i} \frac{F}{R T_{0}} D_{i} C_{i} \frac{\partial \varphi}{\partial x}-D_{i} \frac{\partial C_{i}}{\partial x}, \quad i=1, \ldots, 4 \\
\frac{\partial^{2} \varphi}{\partial x^{2}}=-\frac{F}{\varepsilon_{r}}\left(z_{1} C_{1}+z_{1} C_{2}+z_{3} C_{3}+z_{4} C_{4}\right) \\
R_{1}=R_{2}=0, R_{3}=R_{4}=k_{d} C_{H_{2} O}-k_{r} C_{3} C_{4}=k_{r}\left(k_{w}-C_{3} C_{4}\right) \\
z_{1}=1, z_{2}=-1, z_{3}=1, z_{4}=-1 \\
I=F \sum_{i=1}^{4} j_{i}
\end{gathered}
$$

Here (1) - the material balance equations, (2) - the Nernst-Planck equations for the flows of potassium $\left(\mathrm{K}^{+}, i=1\right)$, chlorine $\left(\mathrm{Cl}^{-}, i=2\right)$, hydrogen $\left(\mathrm{H}^{+}, i=3\right)$ and hydroxyl $\left(\mathrm{OH}^{-}, i=4\right),(3)-$ the Poisson equation for the electric field potential, (4) - formulas describing the dissociation/recombination reaction of water molecules, (5) - the current flow equation, which means that the current flowing through the cross-section of the desalination channel is determined by the ion flow, $\varepsilon_{a}$ - the permittivity of the solution, $F$ - Faraday number, $R$ - universal gas constant, $\varphi$ - potential, $E=-\frac{\partial \varphi}{\partial x}-$ electric field strength, $C_{i}, j_{i}, D_{i}$ - concentration, flow, diffusion coefficient of the i-th ion, $I_{c}-$ is the current density determined by the ion flow.

Boundary conditions:

$$
\begin{gathered}
\left.\left(-\frac{F}{R T} C_{1} D_{1} \frac{\partial \varphi}{\partial x}-D_{1} \frac{\partial C_{1}}{\partial x}\right)\right|_{x=0}=0 \\
C_{2}(t, 0)=C_{2 a} \\
\left.\left(-\frac{F}{R T} C_{3} D_{3} \frac{\partial \varphi}{\partial x}-D_{3} \frac{\partial C_{3}}{\partial x}\right)\right|_{x=0}=j_{3 a} \\
C_{4}(t, 0)=C_{4 a} \\
\varphi(t, 0)=\Delta_{r} \varphi \\
C_{1}(t, H)=C_{1 k} \\
\left.\left(\frac{F}{R T} C_{2} D_{2} \frac{\partial \varphi}{\partial x}-D_{2} \frac{\partial C_{2}}{\partial x}\right)\right|_{x=H}=0 \\
C_{3}(t, H)=C_{3 k}
\end{gathered}
$$




$$
\begin{gathered}
\left.\left(\frac{F}{R T} C_{4} D_{4} \frac{\partial \varphi}{\partial x}-D_{4} \frac{\partial C_{4}}{\partial x}\right)\right|_{x=H}=j_{4 k} \\
\varphi(t, H)=0
\end{gathered}
$$

In this study, the ideal selectivity of ion-exchange membranes is assumed $(6,12)$. The values of ion concentrations passing through the membranes at the boundary points are equal to the values of the exchange capacities of ion-exchange membranes. Theoretically, the exchange capacity of the membrane changes with time, but for a small period of time, this change can be ignored.

The potentiostatic mode is considered - the potential jump $\varphi(t, 0)=\Delta_{r} \varphi$ is considered time-independent. Since the non-catalytic reaction of dissociation/recombination of water molecules is considered, it is assumed that there is no injection of hydrogen and hydroxyl ions from the membrane surface, $j_{3 a}=j_{4 k}=0$.

The initial conditions are taken to be consistent with the boundary conditions as much as possible:

$$
\begin{gathered}
C_{1}(0, x)=C_{2}(0, x)=C_{10}(x)=C_{20}(x) \\
C_{3}(0, x)=C_{4}(0, x)=0 \mathrm{~mol} / \mathrm{m}^{3} \\
\varphi(0, x)=0
\end{gathered}
$$

\section{Research results}

The numerical solution of the boundary value problem is carried out by the finite element method. A large number of calculations were performed for different values of input parameters: boundary concentrations of counterions, potential jump, and initial conditions.

The results are given below, unless otherwise specified, for the following parameters:

$$
\begin{aligned}
& \Delta_{r} \varphi=1.5 \mathrm{~V}, \quad C_{1 k}=C_{2 a}=0.1 \mathrm{~mol} / \mathrm{m}^{3}, \quad C_{3 k}=C_{4 a}=0.01 \mathrm{~mol} / \mathrm{m}^{3}, \\
& C_{1}(0, x)=C_{2}(0, x)=0.1 \mathrm{~mol} / \mathrm{m}^{3}, C_{10}(x)=C_{20}(x)=0 \mathrm{~mol} / \mathrm{m}^{3}, \varphi_{0}(x)=0 .
\end{aligned}
$$

Numerical analysis shows that stands out five qualitatively different periods: a relatively quick transition with duration less than one second, the second period lasts from several seconds to several minutes before the start of the breakdown space charge, quick process breakdown space charge within several seconds, a relatively slow stabilization process of reaching the stationary process taking from several minutes to tens of minutes and, of course, a stationary process.

For numerical analysis, we introduce the following functions:

1) $\frac{1}{F} \rho=z_{1} C_{1}+z_{1} C_{2}+z_{3} C_{3}+z_{4} C_{4}$ is the charge distribution density normalized to the Faraday number. At points where this function is zero, local electroneutrality is observed.

2) $k_{w}-C_{3} C_{4}$ - the equilibrium function of the dissociation/recombination reaction of water molecules. In the region where this function is positive, dissociation prevails over 
recombination, and, conversely, if it is negative, recombination dominates, and in the case of zero, the balance between dissociation and recombination is observed.

\subsection{Features of the transport in the second period}

In the second period, near ion-exchange membranes (AEM and CEM) space charge regions are formed $\left[0, x_{3}\right),\left(x_{9}, H\right]$ (see Figure 2a and Figure 2c) similar to those described in studies [1,2], since $\Delta_{r} \varphi=1.5 \mathrm{~V}$ correspond to the overlimiting mode. Near AEM space charge is negative, and near CEM - is positive charge. These regions consist of quasiequilibrium regions of space charge $\left[0, x_{1}\right),\left(x_{11}, H\right]$ (Figure 2a, Figure 2c.), and extended regions of space charge $\left(x_{1}, x_{3}\right),\left(x_{9}, x_{11}\right)$ (Figure 2a, Figure 2c), according to the terminology of the studies $[1,2]$. In these regions, clearly defined local minimum and maximum of the space charge value are observed at points $x_{1}, x_{2}, x_{10}, x_{11}$ (Figure 2c), the physical causes of which are explained in study [3]. A double electric layer is formed in the middle part of the channel section. As a rule, a double electric layer is formed at the interface due to the difference in the electrical properties of the phases [7]. In this case, the formation of a double electric layer in a homogeneous medium is associated with a recombination reaction. As can be seen from Figure2e, dissociation dominates recombination almost in the space charge region near AEM and CEM, since the electric field strength is significant (Figure $2 \mathrm{~g}$ ) and therefore the electric field pulls apart the hydrogen and hydroxyl ions without allowing them to recombine. Hydroxyl ions are carried by AEM, and hydrogen ions are carried by CEM (see Figure1) the exception is small neighborhoods to the left of the point $x_{3}$ and to the right $x_{9}$, which is due to a significant decrease in the values of the electric field strength (Figure $2 \mathrm{~g}$ ), as a result of which the equilibrium is established. Hydrogen ions formed in the space charge region near AEM are transported to the cathode, and hydroxyl ions formed in the space charge region near CEM are transported to the anode. They occur in the middle part of the channel section and recombine. Equilibrium in the dissociation/recombination reaction can also occur when the concentrations of hydrogen and hydroxyl ions differ greatly, for example, in the vicinity of points and $x_{3}, x_{9}$ (Figure2a and Figure2e). However, to shift the reaction towards recombination, it is necessary that the concentrations of hydrogen and hydroxyl ions are comparable, and the most intense recombination occurs when they are equal, which occurs at the same point $x_{6}$ (compare Figure2a and Figure 2e). There is an excess of hydrogen ions to the left of the point $x_{6}$, and hydroxyl ions to the right of the point. At the point $x_{6}$, the concentrations of potassium and chlorine ions are also equal. That is, at this point there is a local electroneutrality and it separates the positively and negatively charged regions. These regions have local maximum and minimum of space charge values (Figure 2.c), located slightly to the left and right of the point $x_{6}$. Comparison of Figure $2 \mathrm{c}$ and Figure $2 \mathrm{e}$ show that the recombination region is smaller than the regions of the double electric layer and is entirely located inside the latter. To the left of the point $x_{6}$, the concentration of hydroxyl ions is negligible, as is the concentration of hydrogen ions to the right of the point $x_{6}$. Due to the small difference in the diffusion coefficients of hydrogen and hydroxyl ions, the electric field strength at this point has a local maximum (Figure $2 \mathrm{~g}$ ). 
a)

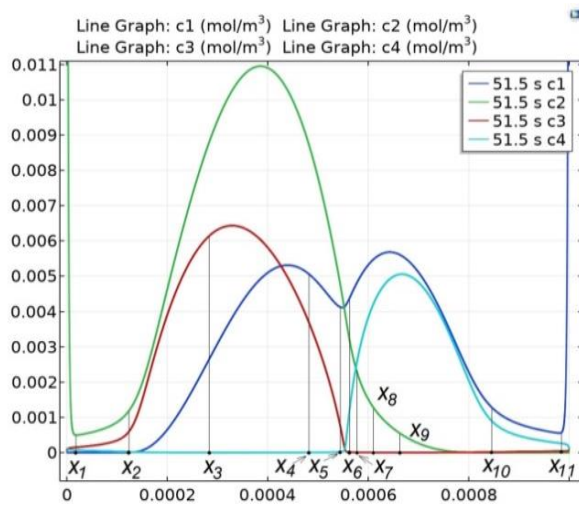

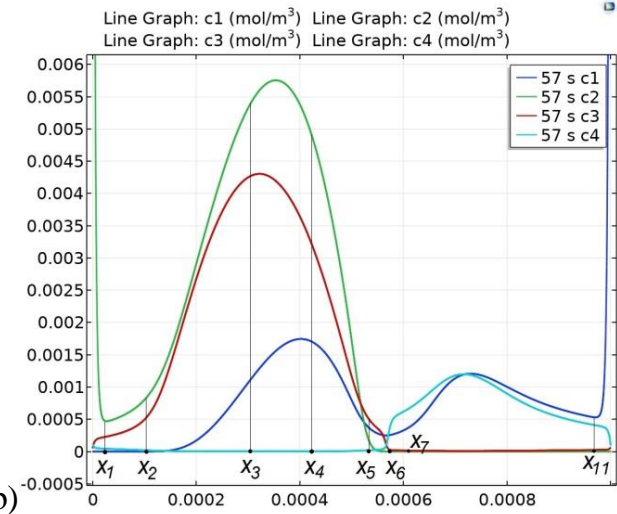
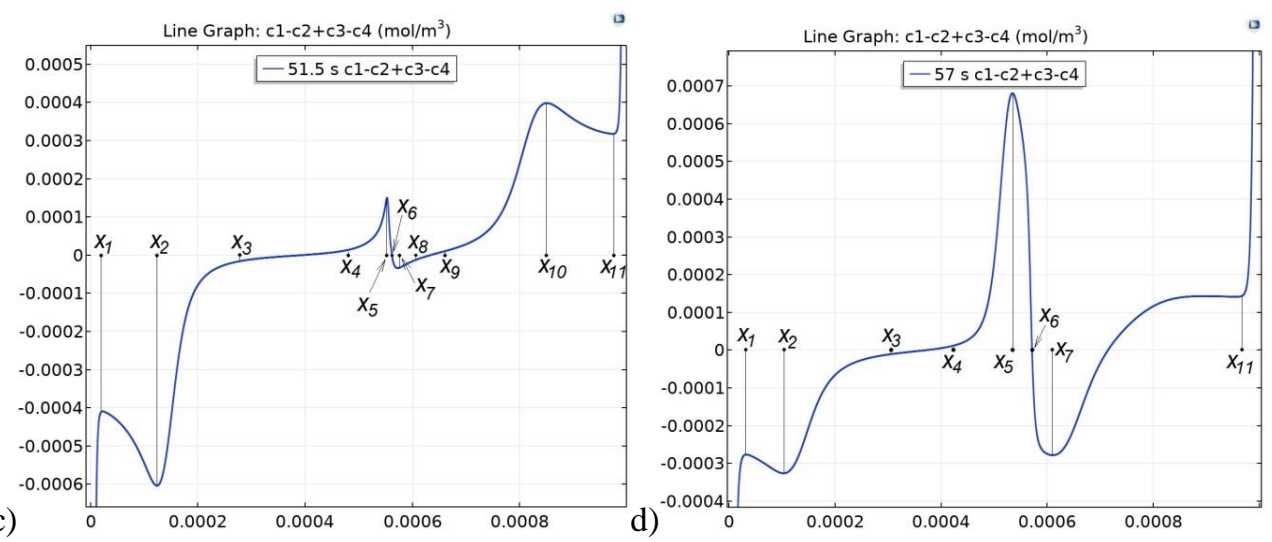

c)
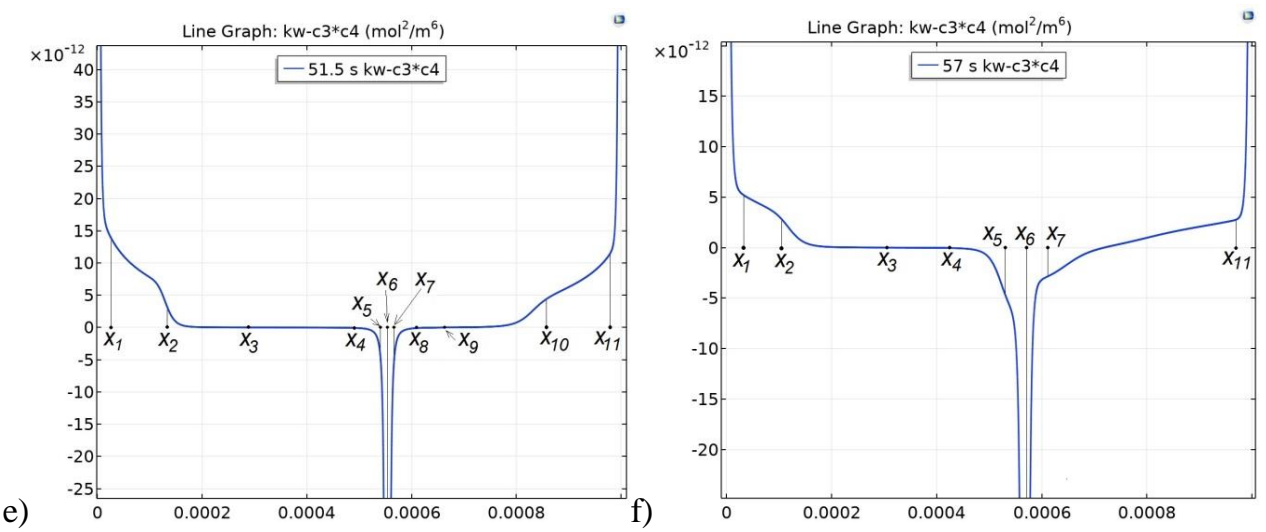
g)

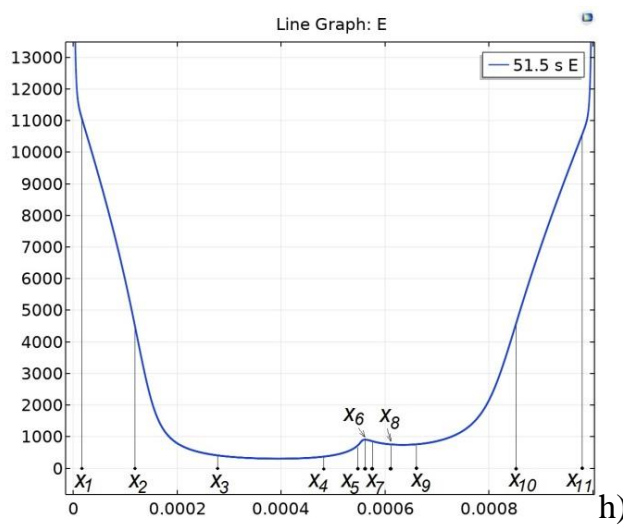

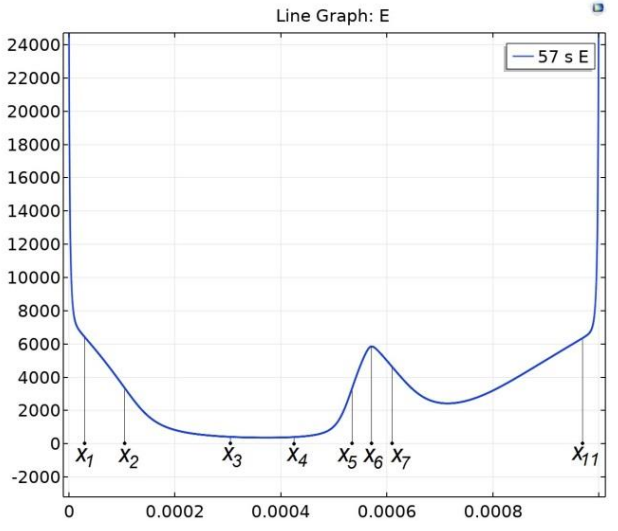

Fig. 2. The process of the salt ions transport with the dissociation/recombination reaction.

Where the left column corresponds to the system condition before the breakdown of space charge, the right - to early breakdown. Points $x_{1}$ and $x_{11}$ - local maximum of the concentration profiles of chlorine and potassium; $x_{2}$ and $x_{10}$-local minimum and maximum of the space charge moving with time deep into the channel section from the ionexchange membranes; $\left(x_{3}, x_{4}\right)$ and $\left(x_{8}, x_{9}\right)$ - intervals of local electroneutrality; $x_{5}$ and $x_{7}$ - local maximum and minimum of the space charge in the middle of the channel section formed due to the dissociation/recombination reaction of water molecules; $x_{6}-$ the point of electroneutrality in the interval $\left(x_{5}, x_{7}\right)$.

\subsection{Space charge breakdown}

As time increases, both the size of the double electric layer and the space charge density in the middle part of the channel cross-section increase significantly. At the same time, the space charge regions near AEM and CEM increase and expand and begin to move towards the middle of the channel section.

\subsubsection{Space charge breakdown near CEM}

Due to the fact that the diffusion coefficient of hydroxyl ions is less than the diffusion coefficient of hydrogen ions, the space charge breakdown begins at CEM. The area of electroneutrality is located at point $x_{9}$ disappears (Figure2c), the negative region of the electrical double layer on the positive space charge region near the CEM and they begin to neutralize: removes the point of maximum $x_{10}$ positive charge and the left quasiequilibrium space charge is formed at the CEM region of electroneutrality (Figure 2d). At the same time, dissociation of water molecules continues in the quasi-equilibrium region of the space charge (Figure 2f). In this case, hydrogen ions are transported through the CEM (Figure 1) and practically do not enter the solution, their concentration is almost zero (Figure 2b). Hydroxyl ions enter the solution, and the absolute value of the local minimum of the negatively charged part of the double layer increases. The point of the local minimum itself begins to shift to the right (Figure 3c). In this case, the previously formed 
region of electroneutrality of the negatively charged part of the double electric layer and the quasi-equilibrium region of the space charge in CEM gradually disappears (Figure 3c) and a double electric layer is formed near CEM in solution. In addition, a positive space charge with a local maximum point $x_{5}$ is preserved in the middle part of the channel section. The breakdown time is about several seconds.

\subsubsection{A first stage of space charge breakdown near AEM}

Due to the fact that the diffusion coefficient of hydrogen ions is greater than that of hydroxyl ions, the density of the positive space charge in the double electric layer in the middle part of the channel section is greater than the density of the negative space charge, the breakdown near AEM occurs somewhat differently, in two stages. At the first stage, the breakdown occurs in the same way as near CEM. As a result, the local minimum at the point $x_{2}$ disappears, a large region $\left(x_{1}, x_{4}\right)$ is appears, where the space charge is so small that it can be considered (but is not) electroneutral, and we get the space charge distribution as in Figure 3c. In this region, the equilibrium condition of Figure $3 d$ is met, and the values of the electric field strength are small. The breakdown time of the first stage is about few seconds.

\subsubsection{Second stage of space charge breakdown near AEM or solution stabilization}

The second stage of breakdown near AEM lasts much longer than the first one, and at the end of it, it goes to a stationary mode. The second stage begins with the movement to the left of a positively charged region with a local maximum point $x_{5}$, both with the movement of the recombination region to the left (Figure $3 \mathrm{e}$ and Figure 3f), and with the continued flow of hydrogen ions from the quasi-equilibrium space charge region near AEM (Figure $3 \mathrm{a}$ and Figure $3 \mathrm{~b}$ ).

a)

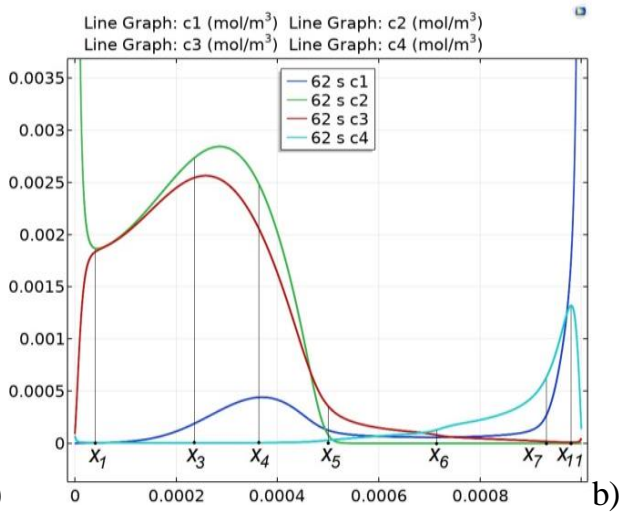

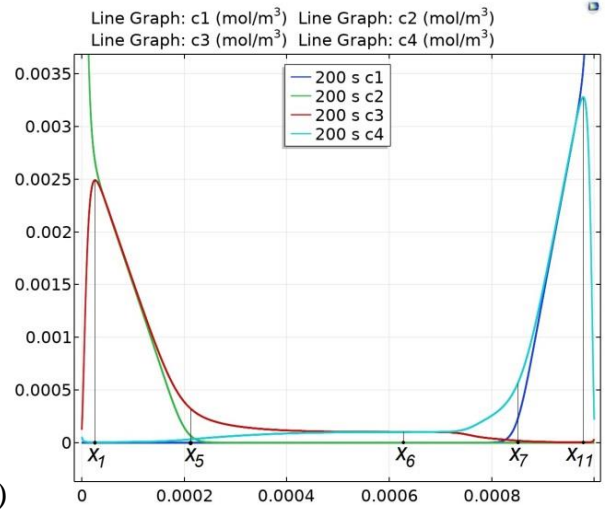


c)
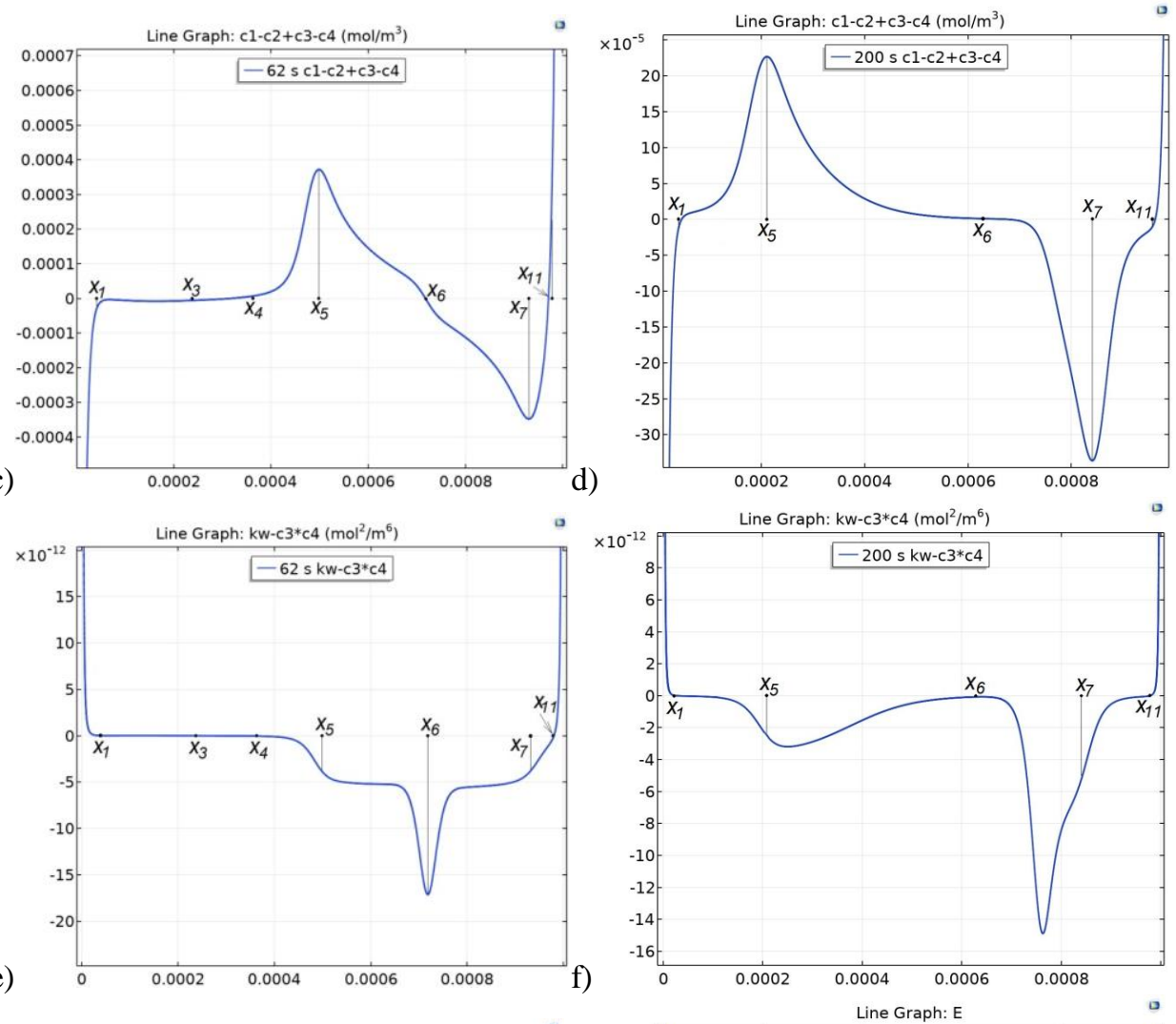

e)
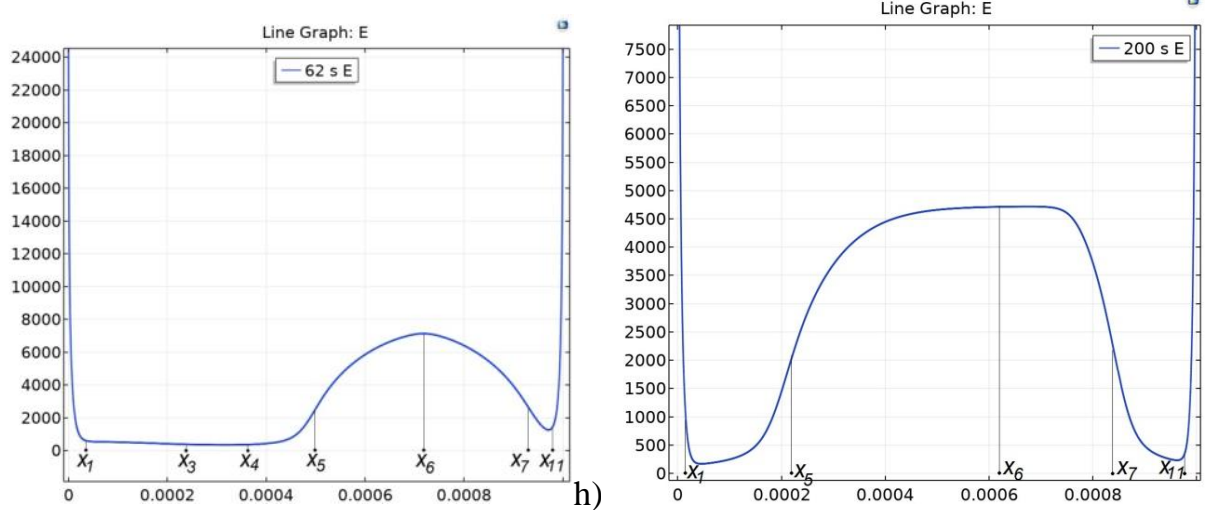

Fig. 3. Space charge breakdown.

Where: the left column corresponds to the end of the breakdown process and the beginning of the stabilization process, and the right column corresponds to the stationary state of the process, which begins with a time $t=109 \mathrm{~s}$. Calculations for comparison were made up to $200 s$ and the difference is within the rounding error range. 


\subsection{Stationary state}

The stationary state starts (as shown in Figure 3) at the given data from about 109 seconds. Near AEM there is a negatively charged space charge region on interval $\left(0, x_{1}\right)$ - Figure $3 \mathrm{~b}$ and to the right $-\mathrm{a}$ positively charged region of space charge - interval $\left(x_{2}, x_{4}\right)$ with local maximum at the point $x_{3}$, which, taking into account the external electric field, compensate for each other and equilibrium occurs. Similarly, a positive space charge region $\left(x_{7}, H\right)$ also appears near the CEM, and a negatively charged space charge region $\left(x_{5}, x_{7}\right)$ with a local minimum point $x_{6}$ appears to the left. In the interval $\left(0, x_{1}\right)$, the concentration of chlorine ions is significantly higher than the concentration of other ions, but in this interval, the concentration of hydrogen ions increases sharply, starting from small values to values comparable to the concentration of chlorine ions. In the interval $\left(x_{1}, x_{4}\right)$, the concentration of hydrogen ions is greater than the concentration of all other ions. In the interval $\left(x_{3}, x_{6}\right)$, the concentration of hydrogen ions and hydroxyl ions has the same order, and in the interval $\left(x_{4}, x_{5}\right)$ they are approximately the same. This leads to the dominance of the recombination reaction over dissociation (see Figure 3c), since this increases the probability of meeting hydrogen and hydroxyl ions, and, accordingly, recombination. The exception is the interval $\left(x_{4}, x_{5}\right)$ where, despite the fact that the concentrations of hydrogen and hydroxyl ions are approximately the same, there is almost equilibrium and not the dominance of recombination over dissociation. This is due to the fact that in the interval $\left(x_{4}, x_{5}\right)$ the values of the electric field strength (Figure 3d) take so large values that the electric field «pulls apart» the hydrogen and hydroxyl ions, thus increasing dissociation. As a result, almost equilibrium is observed at this interval (Figure $3 \mathrm{c})$.

Comparison of the results of Figure 3 with a potential jump $\Delta_{r} \varphi=3 V$ with the results of calculations for a potential jump $\Delta_{r} \varphi=1.5 \mathrm{~V}$ shows their similarity. The difference is that the interval $\left(x_{4}, x_{5}\right)$ where the equilibrium condition is practically satisfied is sufficiently pronounced at $\Delta_{r} \varphi=3 \mathrm{~V}$ and less pronounced at $\Delta_{r} \varphi=1.5 \mathrm{~V}$. At the same time, near the space charge near the CEM at potential jump $\Delta_{r} \varphi=3 \mathrm{~V}$ almost no equilibrium, and at a potential jump $\Delta_{r} \varphi=1.5 \mathrm{~V}$ it is quite pronounced.

\section{Conclusion}

The paper studies the causes of formation and properties of the local maximum (minimum) of the space charge in membrane systems under overlimiting current regimes. It is shown that local maximum and minimum of the space charge appear due to the limited capacity of ion-exchange membranes for a given potential jump. The fundamental laws of this phenomenon are studied. 


\section{Acknowledgments}

The reported study was funded by RFBR and DFG according to the research project № 2058-12018 NNIO_a

\section{References}

1. Rubinstein I and Shtilman L 1979 J. Chem. Soc.Faraday Trans. 231-246

2. Rubinstein I and Zaltzman B 2017 Physical Review Fluids 2(9)

3. Urtenov M, Chubyr N and GudzaV 2020 Membranes 10189

4. Simons R 1979 Nature, Land 28041

5. Urtenov M Kh, Pisissky AV, Nikonenko V V and Kovalenko A V 2018 Membranes and membrane technologies 8(1) 24

6. Urtenov M A Kh, Kovalenko A V, Seidova N M and Pismenskiy A V 2017 Modern methods in the theory of inverse problems and related issues. abstracts of the allRussian scientific conference 110-112

7. Newman J 1977 Electrochemical system (Mir) p 463 